\title{
Improved outcomes with peritoneal dialysis catheter placement after cardiopulmonary bypass in infants
}

\author{
David M. Kwiatkowski, MD, ${ }^{\mathrm{a}}$ Shina Menon, MD, ${ }^{\mathrm{b}}$ Catherine D. Krawczeski, MD, ${ }^{\mathrm{a}, \mathrm{b}}$ \\ Stuart L. Goldstein, MD, ${ }^{\mathrm{a}, \mathrm{b}}$ David L. S. Morales, MD, ${ }^{\mathrm{a}}$ Alistair Phillips, MD, ${ }^{\mathrm{a}}$ Peter B. Manning, MD, ${ }^{c}$ \\ Pirooz Eghtesady, MD, PhD, ${ }^{\mathrm{c}}$ Yu Wang, MS, ${ }^{\mathrm{a}}$ David P. Nelson, MD, PhD, ${ }^{\mathrm{a}}$ and David S. Cooper, $\mathrm{MD}^{\mathrm{a}}$
}

\begin{abstract}
Background: Acute kidney injury (AKI) is common in infants after cardiopulmonary bypass and is associated with poor outcomes. Peritoneal dialysis improves outcomes in adults with AKI after bypass, but pediatric data are limited. This retrospective case-matched study was conducted to determine if the practice of peritoneal dialysis catheter (PDC) placement during congenital heart surgery is associated with improved clinical outcomes in infants at high risk for AKI.
\end{abstract}

\begin{abstract}
Methods: Forty-two infants undergoing congenital heart surgery with planned PDC placement $(\mathrm{PDC}+$ ) were age-matched to infants undergoing similar surgery without PDC placement (PDC-). Demographic, baseline and outcome data were compared. Our primary outcome was negative fluid balance on postoperative days 1 to 3. Secondary outcomes included time to negative fluid balance, time to extubation, frequency of electrolyte corrective medications, inotrope scores, and other clinical outcomes.
\end{abstract}

Results: Baseline data did not differ between groups. The PDC + group had a higher percentage of negative fluid balance on postoperative days 1 and $2(57 \%$ vs $33 \%, P=.04 ; 85 \%$ vs $61 \%, P=.01)$. The PDC+ group had shorter time to negative fluid balance ( 16 vs 32 hours, $P<.0001$ ), earlier extubation ( 80 vs 104 hours, $P=.02$ ), improved inotrope scores $(P=.04)$, and fewer electrolyte imbalances requiring correction $(P=.03)$. PDCrelated complications were rare.

Conclusions: PDC use is safe and associated with earlier negative fluid balance and improved clinical outcomes in infants at high risk for AKI. Routine PDC use should be considered for infants undergoing cardiopulmonary bypass. Further prospective studies are essential to prove causative effects of PDC placement in this population. (J Thorac Cardiovasc Surg 2015;149:230-6)

See related commentary on pages $237-8$.

Acute kidney injury (AKI) is common in infants after surgical repair of complex congenital heart disease, occurring in $30 \%$ to $50 \%$ of patients. ${ }^{1,2}$ Postoperative AKI is associated with significantly increased in-hospital mortality, longer intensive care unit and hospital stays, and prolonged need for mechanical ventilation and inotropic support. ${ }^{2-6}$ Fluid overload has been shown to be an independent predictor of hospital stay, and severity of fluid overload is

From the Heart Institute ${ }^{\mathrm{a}}$ and Center for Acute Care Nephrology, ${ }^{\mathrm{b}}$ Cincinnati Children's Hospital Medical Center, Cincinnati, Ohio; and Division of Cardiothoracic Surgery, ${ }^{\text {c }}$ St Louis Children's Hospital, St Louis, Mo.

The Heart Institute Research Core and Center for Acute Care Nephrology at Cincinnati Children's Hospital Medical Center supported this study.

Disclosures: Authors have nothing to disclose with regard to commercial support.

David M. Kwiatkowski and Shina Menon contributed equally to this work.

Received for publication July 3, 2013; revisions received Nov 15, 2013; accepted for publication Nov 29, 2013; available ahead of print Feb 4, 2014.

Address for reprints: David M. Kwiatkowski, MD, Cincinnati Children's Hospital Medical Center, Cincinnati, Ohio (E-mail: david.kwiatkowski@cchmc.org).

$0022-5223 / \$ 36.00$

Copyright (c) 2015 by The American Association for Thoracic Surgery

http://dx.doi.org/10.1016/j.jtcvs.2013.11.040 associated with worsening outcomes in children, including mortality. ${ }^{7,8}$

Fluid restriction and diuretics comprise the mainstay of fluid balance management in postoperative cardiac surgery patients, despite the increasing literature suggesting that peritoneal dialysis is a safe and effective alternative. ${ }^{9-12}$ In October 2010, our institution made a systematic practice change to electively place a peritoneal dialysis catheter (PDC) at the time of surgery in infants determined to be high risk for AKI based on cardiac anatomy, age, and expected cardiopulmonary bypass (CPB) time (Table 1). We compared outcomes between infants who had a PDC placed during surgery $(\mathrm{PDC}+)$ and age-matched infants with similar surgeries without the placement of a PDC (PDC-) to determine if PDC placement was associated with improved clinical outcomes and if there were adverse events attributable to PDC placement.

\section{METHODS \\ Participants and Study Design}

We performed a retrospective case-matched cohort study of our surgical experience to determine if clinical outcomes improved with planned placement of a PDC at time of surgery (PDC + patients). Review of our institutional surgical database disclosed 55 infants with congenital heart disease less than 6 months of age who underwent corrective heart surgery 


\section{Abbreviations and Acronyms \\ AKI = acute kidney injury \\ $\mathrm{CICU}=$ cardiac intensive care unit \\ $\mathrm{CPB}=$ cardiopulmonary bypass \\ $\mathrm{ECMO}=$ extracorporeal membrane oxygenation \\ PDC = peritoneal dialysis catheter \\ RACHS $=$ Risk Assessment in Congenital Heart Surgery \\ POD = postoperative day}

with CPB and placement of a PDC between October 2010 and August 2012; these patients define the study group to which controls were matched.

Patients in the study group were matched with the most recent infant who underwent the same procedure without placement of a PDC (PDC - patients) from January 2007 to October 2010. Attempts were made to match neonates (age $<1$ month) within 1 week of age and other infants within 2 weeks of age at the time of surgery. The control pool sizes were small $(<20)$ for specific procedures within age restraints, and propensity matching was not used. Within the single ventricle cohort, the dominant ventricle was matched when possible. Potential matched patients were excluded if they died on the day of surgery $(n=2)$ or were started on other extracorporeal support (eg, extracorporeal membrane oxygenation [ECMO] or continuous renal replacement therapy) immediately after bypass $(n=6)$. Infants who underwent uncommon surgical procedures were excluded due to lack of a control $(n=5)$. The resulting study population comprised 42 infants and their matched controls. The Cincinnati Children's Hospital Medical Center Institutional Review Board approved this study. Additional patient consent was not required as only existing data were reviewed.

\section{Patient Management}

Table 1 depicts the criteria for intraoperative PDC placement at the time of CPB. According to institutional protocol, a PDC was placed at the completion of $\mathrm{CPB}$ in patients deemed at high risk for AKI, including all infants younger than 3 months undergoing any $\mathrm{CPB}$ procedure or infants younger than 6 months undergoing heart transplant or younger than 4 months undergoing tetralogy of Fallot or double outlet right ventricle repair. Patients with prolonged CPB times (>120 minutes) had a PDC placed in the operating room at the surgeon's discretion. Patients had a preoperative consultation with the nephrology service when possible.

The pediatric Tenckhoff PDC (37 cm; Quinton Instrument Company, Bothell, Wash.) was inserted via direct transperitoneal access through a purse-string suture in the peritoneum at the inferior aspect of the sternotomy incision and brought out through a stab incision in the left or right upper quadrant. Routine intraoperative fluid management with continuous ultrafiltration and modified ultrafiltration was used. Use of $\mathrm{CPB}$, regional cerebral perfusion, and deep hypothermic circulatory arrest was case specific and according to standard practice. Infants typically returned to the cardiac intensive care unit (CICU) on a milrinone infusion for afterload reduction and low-dose epinephrine for contractility. Calcium (to enhance contractility) and vasopressin (to increase systemic vascular resistance) were added as necessary based on hemodynamics. Dopamine and dobutamine are rarely used in our CICU.

All patients were managed postoperatively in the CICU. As standard, inputs were restricted to two-thirds maintenance volume during the first 2 postoperative days. Medication administration and ventilator support was at the discretion of the CICU physician team. Without a PDC, typical diuretic management begins with furosemide $1 \mathrm{mg} / \mathrm{kg}$ every 6 hours when adequate hemodynamics are achieved. Chlorothiazide is added on the first postoperative day as necessary. In patients with PDCs, furosemide is occasionally used before oliguria during drainage, and is always used as the patient is transitioned from dialysis to standard diuresis, typically on postoperative day (POD) 3 to 4 . Decisions regarding management and removal of the PDC were made by the CICU team in consultation with the nephrology service. The initial peritoneal dialysis prescription was $10 \mathrm{~mL} / \mathrm{kg}$ 1.5\% Dianeal (Baxter Healthcare, McGaw Park, Ill) with potassium chloride $(\mathrm{KCl}) 2$ to $3 \mathrm{mEq} / \mathrm{L}$ and unfractionated heparin 200 units/L; 5-minute fill, 45-minute dwell, 10-minute drain, continuous via Gesco setup (Utah Medical Products, Inc., Midvale, Utah). Peritoneal dialysis was typically started if urine output was less than $1 \mathrm{~mL} / \mathrm{kg} / \mathrm{h}$ for 4 to 8 hours and otherwise left clamped or to drain at the discretion of the CICU team. In efforts to minimize infection, the peritoneal dialysis system is a closed circuit which is replaced every 72 hours, with minimal handling of the circuit by trained dialysis nurses.

\section{Study Variables}

Baseline data included demographic information, primary cardiac diagnosis, age, surgical weight and length, and preoperative creatinine level. Intraoperative variables included surgical procedure performed and associated Risk Assessment in Congenital Heart Surgery (RACHS)-1 score, CPB time, aortic crossclamp time, intraoperative urine output; and whether deep hypothermic circulatory arrest, regional cerebral perfusion, or modified ultrafiltration were used.

Primary outcome data consisted of fluid balance for the first 3 postoperative days, which was calculated as the difference between all fluid inputs and outputs as obtained from nursing documentation in 8-hour intervals.

Secondary outcome data included the number of 8-hour shifts until a negative fluid balance, time to extubation, fluid overload, days to sternal closure (for patients with delayed sternal closure), CICU and hospital length of stay, and in-hospital mortality. We also recorded all direct charges assigned to patients more than the standard CICU stay and physician charges in the first 7 postoperative days to assess costs and use of resources. To assess renal laboratory abnormalities, we devised a metabolic abnormality score, which was calculated using morning electrolyte levels in samples drawn routinely on PODs 1 to 5. Scores ranged from 0 to 4 and were calculated by adding 1 point for each of the following abnormalities: hypokalemia (potassium $<3 \mathrm{mEq} / \mathrm{L}$ ), hypochloremia (chloride $<90 \mathrm{mEq} / \mathrm{L}$ ), metabolic alkalosis (bicarbonate $>30 \mathrm{mEq} / \mathrm{L}$ ), and azotemia (blood urea nitrogen $>30 \mathrm{mg} / \mathrm{dL}$ ). We also collected data on the number of doses of medications given to correct the metabolic abnormalities for the first 5 PODs $(\mathrm{KCl}$, arginine hydrochloride, and acetazolamide). Fluid overload was determined by net fluid balance (L)/baseline weight $(\mathrm{kg})$, and reported as a percentile.

To assess inotropic support, we determined the total duration of inotropic support in days and recorded the type and dose of inotropes twice daily on PODs 1 to 3 to calculate inotrope scores as determined by the following calculation: inotrope score $=$ dopamine dose $(\mu \mathrm{g} / \mathrm{kg} /$ $\min )+$ dobutamine dose $(\mu \mathrm{g} / \mathrm{kg} / \mathrm{min})+[100 \times$ epinephrine dose $(\mu \mathrm{g} /$ $\mathrm{kg} / \mathrm{min})]+[10 \times$ milrinone dose $(\mu \mathrm{g} / \mathrm{kg} / \mathrm{min})]+[10,000 \times$ vasopressin dose $(\mathrm{U} / \mathrm{kg} / \mathrm{min})]+[100 \times$ norepinephrine dose $(\mu \mathrm{g} / \mathrm{kg} / \mathrm{min})]$ originally validated in this population by Gaies and colleagues. ${ }^{13}$

\section{Data Collection and Management}

Medical records were manually interrogated with assistance from data managers from the Cincinnati Children's Hospital Medical Center Heart Institute Research Core. Outcomes were transferred to a Research Electronic Data Capture (REDCap) database using single data entry with appropriate data cleaning methods before analysis.

\section{Statistical Methods}

The median (25th, 75th percentiles) is reported for continuous variables and the Wilcoxon signed rank test was used for comparison between the $\mathrm{PDC}+$ and PDC - groups. Frequency (percentage) is reported for categorical variables and the McNemar exact test was used for comparison 
TABLE 1. Cincinnati Children's Hospital Medical Center protocol for peritoneal dialysis catheter placement after cardiac surgery

\begin{tabular}{lc}
\hline 1. High-risk patients: PDC & Age $<3$ mo undergoing CPB \\
placement planned & Heart transplant $\leq 6$ mo of age \\
preoperatively & Patients undergoing TOF/DORV \\
& repair, age $\leq 4$ mo \\
2. Moderate-risk patients: PDC & Patients undergoing cardiac \\
placement considered based on & transplant, age $>6$ mo \\
clinical condition in the operating & Patients undergoing TOF/DORV \\
room & repair, age $>4$ mo \\
& CPB time $>120$ min \\
\hline
\end{tabular}

$P D C$, Peritoneal dialysis catheter; $C P B$, cardiopulmonary bypass; $T O F$, tetralogy of Fallot; DORV, double outlet right ventricle.

between the PDC + and PDC - groups. Missing values were excluded from each group in the descriptive summary and excluded in matched pairs during comparisons. Based on previous studies that showed worsening outcomes with $10 \%$ fluid overload, we used this as cut-off for survival analysis $^{8}$ and freedom from fluid overload was performed with the Kaplan-Meier method. All tests were 2-sided. All data were analyzed using SAS 9.3 (SAS Institute, Inc. Cary, NC).

\section{RESULTS}

Of the 55 infants less than 6 months age who had a PDC placed routinely after $\mathrm{CPB}$, we were able to successfully match 42 patients one to one with controls based on age and surgical procedure from the existing surgical database. Of patients less than 1 month old, 31 of $34(91 \%)$ had matches within 1 week of age. Of those more than 1 month old, 6 of $8(75 \%)$ had matches within 2 weeks of age. The dominant ventricle was matched in 10 of $11(91 \%)$ patients with a single ventricle. Table 2 lists the demographics and clinical characteristics of the matched groups. All characteristics, including age, had a similar distribution among the groups.

PDC + patients were $24 \%$ more likely to have a negative fluid balance on PODs 1 and 2 compared with those without a PDC $(P=.04$; Table 3$)$. The median time to achieve negative fluid balance was 16 hours sooner in the PDC + group $(P<.0001)$. Most of the negative fluid balance in PDC + patients was achieved by ultrafiltration from dialysis rather than urine output (Table 3), which is demonstrated by the greater percentage of negative balance despite lower urine output. Kaplan-Meier analysis showed the probability of freedom from $10 \%$ fluid overload was significantly higher in $\mathrm{PDC}+$ patients than in $\mathrm{PDC}-$ patients $(P=.002$; Figure 1). By POD 2, 50\% of PDC- patients had $10 \%$ or higher fluid overload compared with only $17 \%$ of $\mathrm{PDC}+$ patients.

PDC + patients had shorter duration of mechanical ventilation ( 80 vs 104 hours, $P=.02$; Figure 2). PDC + infants also had lower morning inotrope scores on PODs 1 to 3 , although this was only statistically significant on day 1 (5.5 vs $8.5, P=.04)$. Although not statistically
TABLE 2. Baseline demographic and clinical characteristics for PDC + and PDC - patients

\begin{tabular}{|c|c|c|}
\hline & $\mathrm{PDC}+(\mathrm{N}=42)$ & PDC $-(\mathbf{N}=\mathbf{4 2})$ \\
\hline Male, n (\%) & $31(74)$ & $31(74)$ \\
\hline \multicolumn{3}{|l|}{ Race, n (\%) } \\
\hline White & $35(83)$ & $33(79)$ \\
\hline Black & 7 (17) & $6(14)$ \\
\hline Asian & $0(0)$ & $1(2)$ \\
\hline Unknown & $0(0)$ & $2(5)$ \\
\hline Age at surgery, $\mathrm{d}$ & $6(3,9)$ & $5(3,14)$ \\
\hline Weight, $\mathrm{kg}$ & $3.4(3.0,3.8)$ & $3.2(2.5,3.9)$ \\
\hline Length, $\mathrm{cm}$ & $50.5(47.0,52.0)$ & $49.0(46.0,53.0)$ \\
\hline Preoperative $\mathrm{Cr}, \mathrm{mg} / \mathrm{dL}$ & $0.5(0.4,0.5)$ & $0.5(0.4,0.7)$ \\
\hline \multicolumn{3}{|l|}{ RACHS-1 score, n (\%) } \\
\hline 2 & $3(8)$ & $4(10)$ \\
\hline 3 & 7 (17) & $6(15)$ \\
\hline 4 & $17(44)$ & $16(41)$ \\
\hline 5 & $0(0)$ & $1(3)$ \\
\hline 6 & $12(31)$ & $12(31)$ \\
\hline \multicolumn{3}{|l|}{ Surgical procedure, $\mathrm{n}(\%)$} \\
\hline Norwood with BT shunt & $9(21)$ & $9(21)$ \\
\hline Arch augmentation & 7 (17) & $7(17)$ \\
\hline Arterial switch operation & 7 (17) & $7(17)$ \\
\hline Neonatal tetralogy repair & $5(12)$ & $5(12)$ \\
\hline Norwood/Sano & $2(4.5)$ & $2(4.5)$ \\
\hline TAPVR repair & $2(4.5)$ & $2(4.5)$ \\
\hline $\begin{array}{l}\text { Others (including cardiac } \\
\text { transplant, truncus } \\
\text { repair, stage II hybrid) }\end{array}$ & $10(24)$ & $10(24)$ \\
\hline Bypass time, min & $164(133,194)$ & $150(116,186)$ \\
\hline Crossclamp time, $\min$ & $62(36,97)$ & $45(30,86)$ \\
\hline
\end{tabular}

Continuous variables are reported as the median (25th, 75 th percentile). Categorical variables are reported as frequency (percentage). There was no statistically significant difference between the 2 groups for any of the baseline characteristics. $P D C+$, Peritoneal dialysis catheter placed during surgery; $P D C-$, no peritoneal dialysis catheter; $\mathrm{Cr}$, creatinine; RACHS, Risk Assessment in Congenital Heart Surgery; BT, BlalockTaussig; TAPVR, total anomalous pulmonary venous return.

significant, mean time to sternal closure was shorter in the patients with a PDC using unmatched analysis (2.3 vs 3.2 days, $P=.13$ ).

We analyzed the groups for metabolic abnormalities. During the first 5 PODs, the PDC+ group had a lower median cumulative metabolic abnormality score of $3(2,4)$ compared with $4(2,6.5)$ in the PDC- group $(P=.002)$. During this period, PDC + infants received fewer doses of medications to correct electrolyte abnormalities compared with PDC - infants $(P=.03)$.

The median duration of PDC placement was $5(3,7)$ days and the use of the PDCs varied in individual patients. On postoperative return to the CICU, $13(31 \%)$ were started on dialysis, $14(33 \%)$ had the PDC left to drain, and 15 $(36 \%)$ had the PDC clamped. Among the PDC+ infants, $25(60 \%)$ received dialysis at some point and the remaining $17(40 \%)$ had the PDC left to drain. The percentage of patients who had the PDC used for dialysis was highest on POD 1 at $52 \%$ and decreased each subsequent day 
TABLE 3. Comparison of outcome variables between PDC + and PDC - patients

\begin{tabular}{|c|c|c|c|}
\hline Outcome & $\mathrm{PDC}+(\mathrm{N}=\mathbf{4 2})$ & PDC $-(N=42)$ & $P$ value \\
\hline $\begin{array}{l}\text { Time to negative fluid } \\
\text { balance, } \mathrm{h}\end{array}$ & $16(0,24)$ & $32(24,48)$ & $<.0001$ \\
\hline $\begin{array}{l}\text { Negative fluid balance } \\
\text { POD1, n (\%) }\end{array}$ & $24(57)$ & $14(33)$ & .04 \\
\hline $\begin{array}{l}\text { Negative fluid balance } \\
\text { POD2, } \mathrm{n}(\%)^{*}\end{array}$ & $35(85)$ & $25(61)$ & .01 \\
\hline $\begin{array}{l}\text { Negative fluid balance } \\
\text { POD3, n }(\%)^{*}\end{array}$ & $26(65)$ & $19(48)$ & .17 \\
\hline Time to extubation, $\mathrm{h}$ & $80(56,128)$ & $104(48,176)$ & .02 \\
\hline \multicolumn{4}{|l|}{$\begin{array}{l}\text { Average urine output, } \\
\mathrm{ml} / \mathrm{kg} / \mathrm{h}\end{array}$} \\
\hline POD1 & $0.82(0.47,1.40)$ & $0.78(0.35,1.52)$ & .43 \\
\hline POD2 & $1.41(0.79,3.28)$ & $2.98(1.46,4.68)$ & .01 \\
\hline POD3 & $4.16(2.44,5.74)$ & $5.10(3.80,7.03)$ & .02 \\
\hline $\begin{array}{l}\text { Total doses of metabolic } \\
\text { corrective medications } \\
\text { for first } 5 \mathrm{~d}\end{array}$ & $1.5(0,3)$ & $3(1,4)$ & .03 \\
\hline \multicolumn{4}{|l|}{ Morning inotrope score } \\
\hline POD1 & $5.5(4.5,8.5)$ & $8.5(5.5,12.5)$ & .04 \\
\hline POD2 & $5.0(2.5,10.0)$ & $5.3(2.5,10.0)$ & .76 \\
\hline POD3 & $2.5(2.0,5.0)$ & $3.5(0.0,8.0)$ & .12 \\
\hline $\begin{array}{l}\text { Percentage of PDCs used } \\
\text { for dialysis }\end{array}$ & & N/A & N/A \\
\hline $\begin{array}{l}\text { POD0, n/total PDC } \\
\text { in place }(\%)\end{array}$ & $13 / 42(31)$ & & \\
\hline $\begin{array}{l}\text { POD1, n/total PDC } \\
\text { in place }(\%)\end{array}$ & $22 / 42(52)$ & & \\
\hline $\begin{array}{l}\text { POD2, n/total PDC } \\
\text { in place }(\%)\end{array}$ & $18 / 41(44)$ & & \\
\hline $\begin{array}{l}\text { POD3, n/total PDC } \\
\text { in place }(\%)\end{array}$ & $12 / 36(33)$ & & \\
\hline $\begin{array}{l}\text { POD4, n/total PDC } \\
\text { in place }(\%)\end{array}$ & $5 / 29(17)$ & & \\
\hline $\begin{array}{l}\text { POD5, n/total PDC } \\
\text { in place }(\%)\end{array}$ & $2 / 24(8)$ & & \\
\hline $\begin{array}{l}\text { Duration of inotrope } \\
\text { requirement, } d\end{array}$ & $5(3,6)$ & $4(2,7)$ & .83 \\
\hline $\begin{array}{l}\text { Need for peritoneal } \\
\text { drain, } \mathrm{n}(\%)\end{array}$ & N/A & $3(7)$ & N/A \\
\hline CICU stay, d & $8(5,13)$ & $6(5,13)$ & .16 \\
\hline Hospital stay, d & $13.5(8.5,19.5)$ & $12(8,24)$ & .60 \\
\hline Death, n $(\%)$ & $6(14)$ & $3(7)$ & .45 \\
\hline \multicolumn{4}{|c|}{$\begin{array}{l}\text { Continuous variables are reported as the median ( } 25 \text { th, } 75 \text { th percentile). Categorical } \\
\text { variables are reported as the frequency (percentage). PDC+, Peritoneal dialysis } \\
\text { catheter placed during surgery; PDC-, no peritoneal dialysis catheter; POD, } \\
\text { postoperative day; N/A, not applicable; CICU, cardiac intensive care unit; } P D C \text {, } \\
\text { peritoneal dialysis catheter. *41 patients in each group on POD2 and } 40 \text { patients in } \\
\text { each group on POD3 because groups with missing values were excluded in pairwise } \\
\text { fashion from both groups. }\end{array}$} \\
\hline
\end{tabular}

(Table 3). Given the retrospective nature of the study, at time points after initial return to the CICU, we are unable to discern between PDCs that were clamped and those that were left to drain with no output. However, these patients were the minority, totaling less than $10 \%$ on PODs 1 to 3.
To determine the role of dialysis in patients with PDCs, subanalysis was performed for the matched controls versus patients with the PDC used for dialysis and drained separately. An unmatched analysis also compared patients with the PDC left to drain versus PDC used for dialysis. Comparison showed all subgroups performed equally in terms of hours to extubation, time to negative fluid balance, and the proportion of infants who achieved a negative fluid balance. However, this comparison was limited by small sample sizes after the groups were subdivided.

Three patients in the PDC + group had related adverse events. One patient, who was started on ECMO on POD 4, had bloody effluent in the catheter. A second needed to have his catheter replaced because of omental occlusion. The third, and the only significant complication, occurred in a patient who had omental evisceration after catheter removal and needed a partial omentectomy and secondary closure of the abdominal wall, performed at the bedside. Three patients from the PDC- group had peritoneal drains placed postoperatively at the bedside to help with management of fluid balance. There were 9 deaths overall; 6 in the PDC + group and 3 in the PDC - group $(P=.45)$.

The median length of hospital stay was similar in both groups $(P=.60)$. Cost analysis of CICU stay showed that PDC + patients had similar median direct charges of $\$ 2265(1917,2898)$ daily during the first 7 PODs compared with $\$ 2669(1865,3167 ; P>.05)$, suggesting that the additional cost of peritoneal dialysis was offset by decreased cost expenditure elsewhere.

\section{DISCUSSION}

Extravascular fluid retention is a frequent complication after CPB in infants due to AKI, capillary leak, fluid resuscitation, and low cardiac output syndrome decreasing renal perfusion. In our critically ill cohort, balancing fluid restriction and aggressive diuresis with provision of adequate volume to maintain preload and prevent electrolyte imbalances often proves challenging. A persistent positive fluid balance after achieving hemodynamic stability may be harmful in the recovery phase and may negatively affect organ function. Recent studies have shown a relationship between positive fluid balance and cardiorespiratory complications, including impaired pulmonary gas exchange and reduced lung compliance., ${ }^{7,10}$ Increasing degrees of fluid overload may result in fewer ventilator-free days along with longer CICU and hospital length of stays. ${ }^{7,8}$ Although removal or prevention of excess fluid accumulation is universally accepted as a postoperative goal, the most effective means of elimination remains debatable. Practitioners often rely on loop diuretics such as furosemide, which are not uniformly effective, and potentially detrimental, in 

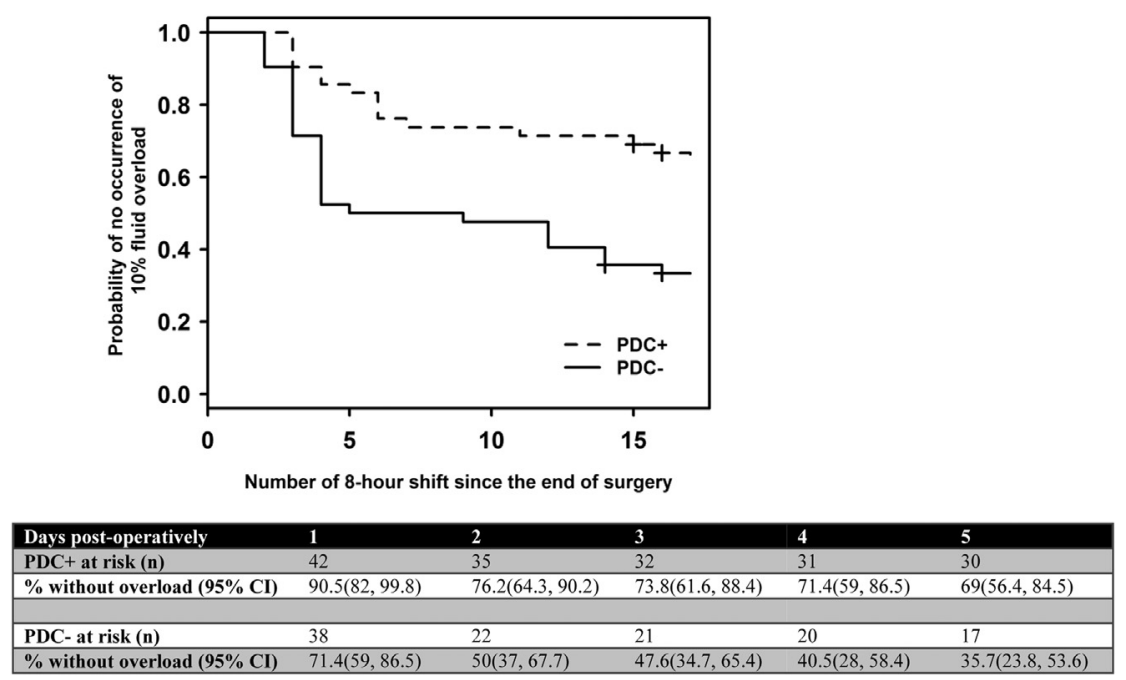

FIGURE 1. Kaplan-Meier curve showing freedom from fluid overload for the PDC group (PDC + ) and the non-PDC group (PDC-). By postoperative day 2 (fifth shift after surgery), only $17 \%$ of patients with a PDC had $10 \%$ or higher fluid overload compared with $50 \%$ from the control group $(P=.002)$. $P D C$, Peritoneal dialysis catheter; $P D C+$, peritoneal dialysis catheter placed during surgery; $P D C-$, no peritoneal dialysis catheter.

patients with AKI. ${ }^{11,12}$ An adult randomized trial showed that furosemide use after CPB was associated with a 2 -fold increase in postoperative creatinine levels and rate of AKI was significantly higher. ${ }^{14}$

In this study of infants undergoing $\mathrm{CPB}$, elective placement of a PDC at the time of surgery was associated with improved clinical outcomes. These infants were more likely to have a negative fluid balance in the first critical PODs and a shorter time to a negative fluid balance. Our study demonstrates that patients with a PDC are more likely to avoid $10 \%$ fluid overload (Figure 1), which has been demonstrated to be an independent risk factor for death in critically ill infants and children. ${ }^{8}$ Most of the

\section{Clinical Outcomes}

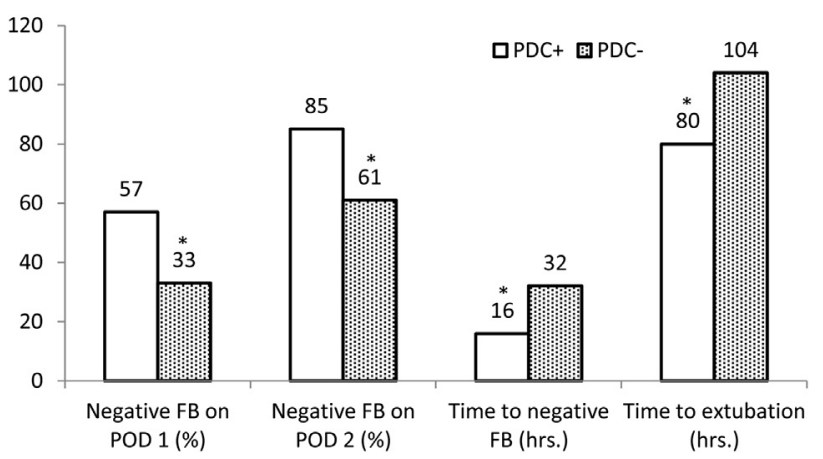

FIGURE 2. Bar plot showing difference in outcomes between the 2 groups. *All comparisons in the bar plot are significant $(P<.05) . P D C$, Peritoneal dialysis catheter; $P O D$, postoperative day; $F B$, fluid balance; $P D C+$, peritoneal dialysis catheter placed during surgery; $P D C-$, no peritoneal dialysis catheter. negative fluid balance in our PDC + patients was achieved by ultrafiltration.

The patients in the PDC + group were successfully extubated 24 hours sooner, an important marker of critical care deintensification. Early extubation is desirable for several reasons, including decreased rates of ventilatorassociated pneumonia, less need for sedative and paralytic agents, less CICU time, and decreased costs and use of resources. ${ }^{15,16}$ Furthermore, infants with congenital heart disease often have more favorable hemodynamics with spontaneous ventilation, underscoring the value of this outcome within this population.

The PDC + group also demonstrated trends toward earlier sternal closure among the 35 patients who returned to the CICU with an open sternum. Although the sample size was not adequate to prove association, these data corroborate the existing literature, which suggests negative fluid balance is necessary for successful sternal closure. ${ }^{17}$ This is important for minimization of sedative medication use and surgical site infection, as demonstrated by a recent study of 375 patients with delayed sternal closure, which found duration of open sternum was a predictor of surgical site infection. ${ }^{18}$

PDC + patients in this study received fewer electrolyte corrective medications, and had lower metabolic abnormality scores. Postoperative electrolyte balance is desired for optimization of cardiac function and prevention of arrhythmia. Electrolyte imbalance is a proven risk factor for death among infants receiving ECMO in the setting of failed conventional CPR. ${ }^{19}$ A study of adults treated with diuretics for hypertension showed that those with mild hypokalemia (3.0-3.5) were 24.3 times more likely to have 
sudden death. ${ }^{20}$ Diuretic-induced metabolic alkalosis, which was noted in our population, decreases respiratory drive, which can cause atelectasis and delay ventilator weaning. ${ }^{21,22}$

Previously, peritoneal dialysis was reserved for patients who developed AKI and fluid overload who did not respond to fluid restriction and diuretics. ${ }^{23-25}$ However, early initiation of peritoneal dialysis, particularly in infants at high risk of developing $\mathrm{AKI}$ after $\mathrm{CPB}$, is now widely used to prevent fluid overload. ${ }^{5,9,10,26}$ This study adds to the existing literature on other populations; early peritoneal dialysis results in a better fluid balance and fewer days on the ventilator without increasing complications.

As expected with a relatively small sample size and low incidence of mortality, a survival difference was not seen. The association of early peritoneal dialysis with decreased mortality has been reported in a cohort of 146 infants on peritoneal dialysis for AKI after CPB by Bojan and colleagues. ${ }^{5}$ This group demonstrated that dialysis started within the first POD was associated with a $46 \%$ decrease in 30-day mortality compared with delayed dialysis. Their report differs from the current study by focusing on the subset of patients who had already developed AKI to the degree they required renal replacement therapy, thus constituting a more ill cohort.

Despite earlier extubation and lower inotrope scores, we did not see a significant difference in the duration of CICU stay between the 2 groups. Factors independent of patient recovery, including seasonality, hospital logistics, and bed availability outside the CICU may affect the length of stay. ${ }^{2}$ We found that PDC placement and dialysis did not increase the cost of hospital stay. The additional cost of performing peritoneal dialysis was offset by a decrease in other costs including respiratory therapy, laboratory charges, and medicines. In the face of increasing health care costs, it is noteworthy that peritoneal dialysis improves outcomes without adding to costs.

Among the PDC- patients, 3 of $42(7 \%)$ had late placement of peritoneal drains. Although no complications were seen, this represents a higher-risk practice, because infants who require bedside procedures need intervention with limited sterility in the context of clinical deterioration with worsening ascites and renal insufficiency. The literature suggests that bedside procedures are more prone to infection, exit site leakage, and mechanical injury. ${ }^{10}$

In the PDC + group, 3 patients had complications attributable to the catheter. This was early in our surgical experience and our placement/removal technique has since been modified. Two complications were relatively minor and none affected the ultimate outcome. No patient had peritonitis. This and other studies report low complication rates associated with PDC, which is largely attributed to intraoperative placement of the catheter under direct visualization with sterile fields. ${ }^{10,27}$ The most common complications noted in previous studies have been positive peritoneal culture, bowel injury, hyperglycemia, and hemodynamic instability with drainage. ${ }^{28}$ Multiple techniques of catheter placement have been described including indirect or direct transperitoneal and transdiaphragmatic approaches, with varying rates of complications. ${ }^{29}$ Our institution uses uncuffed catheters placed by a direct transperitoneal method. Intraoperative catheter placement under direct visualization decreases the likelihood of the infectious and mechanical complications that were described in early use of peritoneal dialysis. Our low complication rate can also be attributed to the use of a closed circuit, which is replaced every 72 hours, low dwell volumes $(10 \mathrm{~mL} / \mathrm{kg})$, and minimal handling of the circuit by trained dialysis nurses. ${ }^{30}$

\section{Limitations}

Although this is the largest study to compare outcomes between patients with a PDC and matched controls, it is subject to traditional bias inherent to studies with a retrospective cohort design. We attempted to avoid bias by matching each patient with a control of similar age undergoing the same procedure. The method for matching was defined a priori. Because patient groups were from different periods of time, they may have been influenced by changes in physician practices, particularly attitudes toward diuretic use and the timing of extubation. However, our study suggests that patients in the PDC + group achieved a negative fluid balance from ultrafiltration and not aggressive diuresis. Although this study is subject to selection bias because the rare sick patient who had a PDC placed before 2010 was excluded from the analysis, this bias strengthens our hypothesis. Unfortunately, our study design makes it difficult to discern whether improved outcomes should be attributed to PDC drainage or dialysis. This question would serve as a future area for study. Our metabolic abnormality score was developed empirically for this study and will need to be validated in future studies.

\section{CONCLUSIONS}

Elective PDC placement at the time of CPB in infants at high risk for $\mathrm{AKI}$ is a reasonable practice. We demonstrate that the presence of a PDC allows for more effective fluid management and improved clinical outcomes. These results underscore the need for a prospective randomized trial to determine the causal relationship between peritoneal dialysis and clinical outcomes.

\section{References}

1. Krawczeski CD, Woo JG, Wang Y, Bennett MR, Ma Q, Devarajan P. Neutrophil gelatinase-associated lipocalin concentrations predict development of acute 
kidney injury in neonates and children after cardiopulmonary bypass. J Pediatr. 2011;158:1009-15.e1.

2. Blinder JJ, Goldstein SL, Lee VV, Baycroft A, Fraser CD, Nelson D, et al. Congenital heart surgery in infants: effects of acute kidney injury on outcomes. J Thorac Cardiovasc Surg. 2012;143:368-74.

3. Dasta JF, Kane-Gill SL, Durtschi AJ, Pathak DS, Kellum JA. Costs and outcomes of acute kidney injury (AKI) following cardiac surgery. Nephrol Dial Transplant. 2008;23:1970-4.

4. Zappitelli M, Bernier PL, Saczkowski RS, Tchervenkov CI, Gottesman R, Dancea A, et al. A small post-operative rise in serum creatinine predicts acute kidney injury in children undergoing cardiac surgery. Kidney Int. 2009;76: 885-92.

5. Bojan M, Gioanni S, Vouhe PR, Journois D, Pouard P. Early initiation of peritoneal dialysis in neonates and infants with acute kidney injury following cardiac surgery is associated with a significant decrease in mortality. Kidney Int. 2012; $82: 474-81$.

6. Hazle MA, Gajarski RJ, Yu S, Donohue J, Blatt NB. Fluid overload in infants following congenital heart surgery. Pediatr Crit Care Med. 2013;14: 44-9.

7. Arikan AA, Zappitelli M, Goldstein SL, Naipaul A, Jefferson LS, Loftis LL. Fluid overload is associated with impaired oxygenation and morbidity in critically ill children. Pediatr Crit Care Med. 2012;13:253-8.

8. Sutherland SM, Zappitelli M, Alexander SR, Chua AN, Brophy PD, Bunchman TE, et al. Fluid overload and mortality in children receiving continuous renal replacement therapy: the prospective pediatric continuous renal replacement therapy registry. Am J Kidney Dis. 2010;55:316-25.

9. Picca S, Ricci Z, Picardo S. Acute kidney injury in an infant after cardiopulmonary bypass. Semin Nephrol. 2008;28:470-6.

10. Sorof JM, Stromberg D, Brewer ED, Feltes TF, Fraser CD Jr. Early initiation of peritoneal dialysis after surgical repair of congenital heart disease. Pediatr Nephrol. 1999;13:641-5.

11. Bagshaw SM, Delaney A, Haase M, Ghali WA, Bellomo R. Loop diuretics in the management of acute renal failure: a systematic review and meta-analysis. Crit Care Resusc. 2007:9:60-8.

12. Ho KM, Power BM. Benefits and risks of furosemide in acute kidney injury. Anaesthesia. 2010;65:283-93.

13. Gaies MG, Gurney JG, Yen AH, Napoli ML, Gajarski RJ, Ohye RG, et al. Vasoactive-inotropic score as a predictor of morbidity and mortality in infants after cardiopulmonary bypass. Pediatr Crit Care Med. 2010;11: 234-8.

14. Lassnigg A, Donner E, Grubhofer G, Presterl E, Druml W, Hiesmayr M. Lack of renoprotective effects of dopamine and furosemide during cardiac surgery. J Am Soc Nephrol. 2000;11:97-104.

15. Dries DJ, McGonigal MD, Malian MS, Bor BJ, Sullivan C. Protocoldriven ventilator weaning reduces use of mechanical ventilation, rate of early reintubation, and ventilator-associated pneumonia. J Trauma. 2004; 56:943-51.
16. Alghamdi AA, Singh SK, Hamilton BC, Yadava M, Holtby H, Van Arsdell GS, et al. Early extubation after pediatric cardiac surgery: systematic review, meta-analysis, and evidence-based recommendations. J Card Surg. 2010;25:586-95.

17. Tabbutt S, Duncan BW, McLaughlin D, Wessel DL, Jonas RA, Laussen PC. Delayed sternal closure after cardiac operations in a pediatric population. J Thorac Cardiovasc Surg. 1997;113:886-93

18. Harder EE, Gaies MG, Yu S, Donohue JE, Hanauer DA, Goldberg CS, et al. Risk factors for surgical site infection in pediatric cardiac surgery patients undergoing delayed sternal closure. J Thorac Cardiovasc Surg. 2013;146: 326-33.

19. Raymond TT, Cunnyngham CB, Thompson MT, Thomas JA, Dalton HJ, Nadkarni VM. Outcomes among neonates, infants, and children after extracorporeal cardiopulmonary resuscitation for refractory inhospital pediatric cardiac arrest: a report from the National Registry of Cardiopulmonary Resuscitation. Pediatr Crit Care Med. 2010;11:362-71.

20. Cohen HW, Madhavan S, Alderman MH. High and low serum potassium associated with cardiovascular events in diuretic-treated patients. J Hypertens. 2001; 19:1315-23.

21. Hodgkin JE, Soeprono FF, Chan DM. Incidence of metabolic alkalemia in hospitalized patients. Crit Care Med. 1980;8:725-8.

22. Greenberg A. Diuretic complications. Am J Med Sci. 2000;319:10-24.

23. Chan KL, Ip P, Chiu CS, Cheung YF. Peritoneal dialysis after surgery for congenital heart disease in infants and young children. Ann Thorac Surg. 2003;76: 1443-9.

24. Chien JC, Hwang BT, Weng ZC, Meng LC, Lee PC. Peritoneal dialysis in infants and children after open heart surgery. Pediatr Neonatol. 2009;50: 275-9.

25. Hanson J, Loftness S, Clarke D, Campbell D. Peritoneal dialysis following open heart surgery in children. Pediatr Cardiol. 1989;10:125-8.

26. Picca S, Principato F, Mazzera E, Corona R, Ferrigno L, Marcelletti C, et al. Risks of acute renal failure after cardiopulmonary bypass surgery in children: a retrospective 10-year case-control study. Nephrol Dial Transplant. 1995;10: 630-6.

27. Swan P, Darwish A, Elbarbary M, Halees ZA. The safety of peritoneal drainage and dialysis after cardiopulmonary bypass in children. J Thorac Cardiovasc Surg. 1997; 114:688-9.

28. Werner HA, Wensley DF, Lirenman DS, LeBlanc JG. Peritoneal dialysis in children after cardiopulmonary bypass. J Thorac Cardiovasc Surg. 1997;113:64-8; discussion 68-70.

29. Murala JS, Singappuli K, Provenzano SC Jr, Nunn G. Techniques of inserting peritoneal dialysis catheters in neonates and infants undergoing open heart surgery. J Thorac Cardiovasc Surg. 2010;139:503-5.

30. Valeri A, Radhakrishnan J, Vernocchi L, Carmichael LD, Stern L. The epidemiology of peritonitis in acute peritoneal dialysis: a comparison between open- and closed-drainage systems. Am J Kidney Dis. 1993;21:300-9. 